\title{
Composition Basics and Analysis of Visual Language of the Product
}

\author{
K. MARKOVA \\ Technical University of Varna, Manufacturing, Engineering and Technologies Faculty, Industrial Design \\ Department, kremenacankova@abv.bg
}

Abstract. The analysis of the visual language of the product demands deeper analysis of the user's profile and built behavioral patterns. There is an existing group of factors that have relatively constant nature - gender differentiation, age differentiation, belongingness to specific group - social status, place in hierarchy, race, religion, people with special needs, etc. The analysis of the visual language of the product can be done by analyzing the basic arsenal of the composition - the means of composing form shaping: scale -related to the correct size of the products and their components; proportions -associated with organizing the form plastical and visual order of the whole and its parts; contrast - related to the shape of the product, semantical or bounded to past experience; color-bounded to both formal characteristics and plasticity, as well as to contrast and its meaningful designation; plastic of the form linked to the proportions and scale, which determines not only the structural and formal characteristics of the products, but also the meaningful reading of the forms.

The analysis of the individual composite devices is demonstrated by Ingo Maurer's lighting fixtures.

\section{Introduction}

The problem with the expressiveness of consumer products is characterized with its dynamics. Undoubtedly, the evolution of technology is a leading factor in the dynamics, because it allows the realization of functions and forms unknown in the past, which quickly become an object of consumers' desire. But there are other two major factors as well - the cultural model and the information. The cultural present, with its norms and trends, determines to a great extent the character of the pledged information in the product's look. The free and fast access of information, provided to us by the internet, determines the dynamics of the consumer's behavior and its changing nature. This behavior demands in the utilitarian functions of the products to be included such functions and qualities that provide the potential of the realization of a game situation. So there is a process in which the contemporary culture, perceived more and more like entertainment, is bounded with the stream of information, leading to the saturation of the senses and desires for change so this process founds its image in the visual language of the consumer's products.

\section{Methods and results}

The analysis of the visual language of the product demands deeper and deeper analysis of the user's profile and built behavioral patterns. While this patterns have relatively quickly changing nature, 
based on the changing cultural status quo, there is an existing group of factors that have relatively constant nature (but changing ahead in time). The main ones are:

- Gender

The gender differentiation stakes on relatively stable in time behavioral models which include and distribution over the defined social roles. The conservatism of such models, like "blue for boys, "pink for girls" ensures them vitality in the form of alternative even when the one-sidedness of the model is disturbed.

The main anthropometric differences between the two genders are also in the range of this factor.

- Age differentiation

Extremely sustainable model for analysis. The different age groups have their own specific physical, cognitive, cultural and other specifications, which determine user's behavior and are a major factor for both operational qualities of the products, and the determination of their visual language.

- Belongingness to specific group - social status, place in hierarchy, race, religion, people with special needs, etc.

This factor is engaged with the possibility set in the qualities of the products to be distinguishable for the receiver. In other words, the specific group of users to acknowledge themselves as exclusive users of the product. In this factor, the criteria are very diversified, for example - style, materials, symbolical meaning, price, colors, fashion trends, resistance of the model ("classical" combinations), demonstration of technological novelty, innovations, restrictions imposed in the form of certain canons, physical constraints and many others.

The analysis of the visual language of the product can also be done by analyzing the basic arsenal of the composition, more precisely the means of composing form shaping.

It should be noted here that the term " composing form shaping " in the papers is referred to the utilitary products, for the serial or mass production and not for works of art.

The analysis of the individual composite devices is demonstrated by Ingo Maurer's lighting fixtures, shown in Figures 1 and 2 as follows:
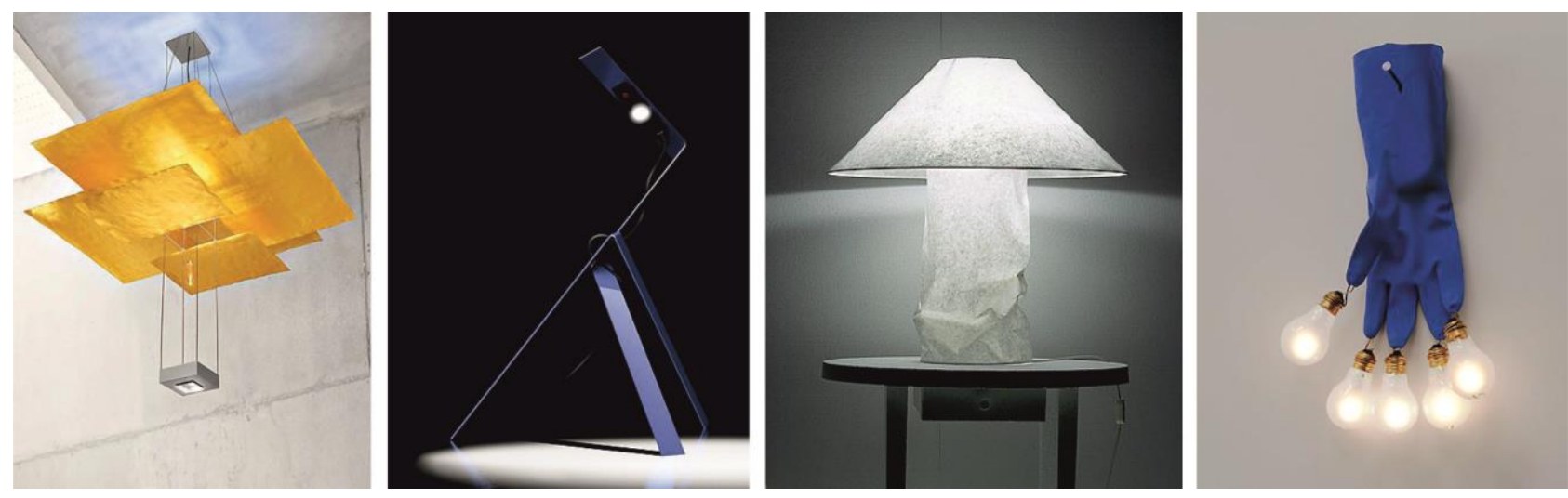

Figure 1. From left to right - „Oh Mei Ma Kabir“ - 1997; „Jetzt 2“ - 2015, an Axel Schmid's lighting fixture replica from 2009; „Lampampe“-1980; „Luzy on the Wall“- 2018. 

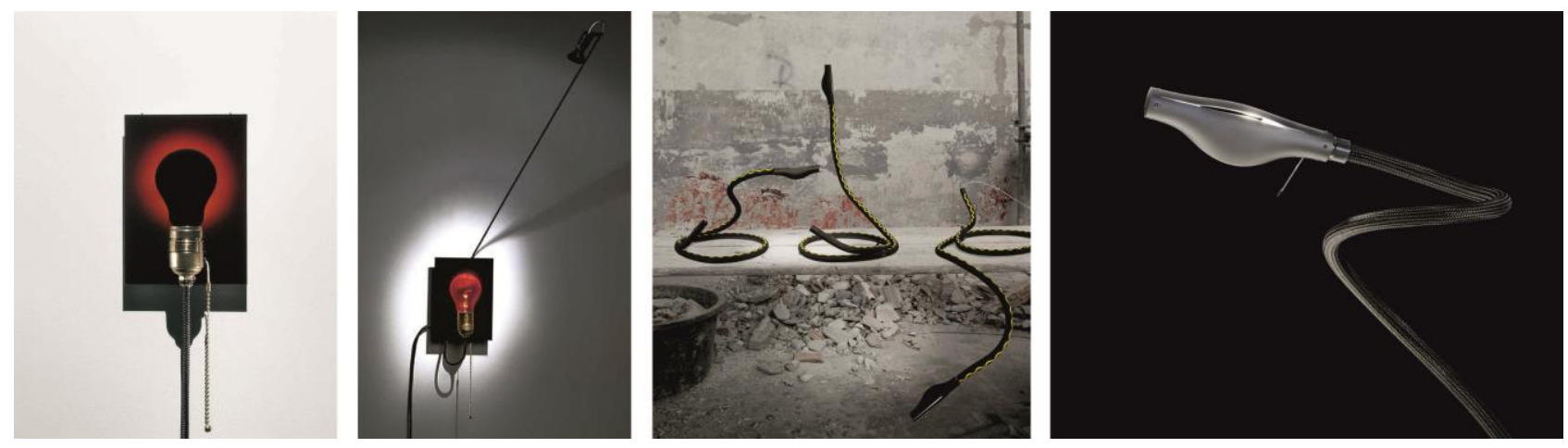

Figure 2. From left to right - „Dead Bulb Alive“ - 2000; „Holonzki“-2000; „Alizz T. Cooper“-2008; „Metall T.

Cooper" -2009.

The main compositional means are:

Scale - It's related to the correct size of the products and their components, in other words - the properly designed dimensions provide operational and visual comfort. The elements that give the most accurate feature about the size of the entire product are called "scale indicators", such as spins, switches, handles, etc. The rubber glove from "Luzy on the Wall" (Figure 1) gives information about the overall size of the product and is a scale indicator.

Proportions - Proportions are associated with organizing the form plastical and visual order of the whole and its parts. They are a selection of specific dimensional relationships that allow the whole to function as one system and ensures physical and visual balance. This balance allows the realization of the operating function of the product.

The handmade gold-coated sheets of "Oh Mei Ma Kabir" (Figure1) are designed in a certain proportional relationship to each other and to the rest of the elements of the object. Thus, they represent not only a harmonic configuration of elements, but also perform the function of a diffuser on the light from above. Of course, their scale complies with both the size of standardized factory components (lamps, connections) and the function of the entire main light source in the room.

The "Jetzt 2" desktop lamp (Figure 1) has the appropriate proportions of a desk lamp $(40 / 30 \mathrm{~cm})$. The total size of the article is coordinated with the numbers and proportional (corresponding) dimensions of the elements of the whole so to provide its functional predestination - targeted, working lighting which is realized through a visually simplified form, in which the ratios between the elements and the whole do not visually or semantically load the user.

The proportions of the two "Alizz T. Cooper" shaping elements (Figure 2) are based on the naturally occurring proportions in the body / head size of poison snakes.

Contrast - A powerful mean of attracting the attention of the addressee. It can be related to the shape of the product - shaping patterns, number of elements, color, materials; may be semantic - to oppose the conceptual content of a physical model; to be bounded to past experience, atavism; to position a particular element in the whole or the whole itself in a specific way, etc.

The form of "Lampampe" (Figure 1) is based on formal and semantic contrast - a simple, symmetrical form of two basic elements, one with the right conical shape, and the other with a regular cylindrical 
shape but disturbed, disrupted. The wrinkled Japanese paper at the base of the product creates a sense of different materiality, something organic, and at the same time raises doubts about the physical stability of the product.

The contrast in "Luzy on the Wall" (Figure 1) is again formal and semantic - the soft, flexible rubber glove contrasts sharply with the metal-glass combination (the five lamps), contrasting in color and tone. Inconspicuous contrast opposes the usual function of the glove (to protect the hands) with its new role (being part of the lighting system); the positioning of the wall also contrasts with the conventional one - no hidden fastener is chosen, but tends to be nailed to the wall, there is an element of aggression, coercion.

Color - It's bounded to both formal characteristics and plasticity, as well as to contrast and its meaningful designation. It can be a mean of manipulating human emotions, for example, to provoke a desire for possession.

In "Oh Mei Ma Kabir" (Figure 1) the color of gold is symbolically bounded to the idea of sunlight. Gilded sheets are also physically positioned as far from the user as they can, they are positioned at the appropriate height so they can not be touched by humans - a second analogy to the sun.

The contrasting color combination in "Alizz T. Cooper" (Figure 2) based on atavism (in nature, the combination of yellow and black is often a warning signal of danger - stinging insects, for example) dramatically alters the nature of the used materials by symbolically designating them.

The color can be related to certain fashion trends or combinations considered to be cultural evergreens - the red-black combination for example ("Dead Bulb Alive" and "Holonzki", Figure 2).

The perceptions of color depend on the features of visual perception and are a powerful factor in the formation of visual memory.

Plasticity. Plastic of the form - A basic tool of form shaping, linked to the proportions and scale, which determines not only the structural and formal characteristics of the products, but also the meaningful reading of the forms. It refers to both the structure, the construction of the shape and the nature of the surface, the coating or the characteristics of the materials used.

This tool often has the basic geometric patterns as the starting point, depending on the specifics of the visual perceptions and the simplicity and clarity of the model.

The simplicity of the visual image of "Jetzt 2" (Figure 1) is achieved not only by the small number of shaping elements, but also by the aesthetics, in which there is nothing unnecessary. The shape of plastic resembles a living organism - it allows a specific meaningful reading.

The overall contour of the lighting-hollograms "Dead Bulb Alive" and "Holonzki" (Figure 2) follows the basic geometric figure - rectangle. The plasticity of the shape is based on the contrast between the correct geometry (contour, circular shape of the holographic image) and the plastic details on the phonon and the breaker (the string). Simple against complicated. 


\section{Conclusions}

The process of analyzing the informational expression of consumer products can be relied on the means of compositional forming. Properly used means provide operational and visual comfort to the users. They provide a specific visual language that can be recognized by different user groups. In this process, the local culturological model also occupies an interactive position.

\section{References}

[1] Ingo-maurer.com < https://www.ingo-maurer.com/en/ >(4.10.2018). 